\title{
Traumatologie anopérinéale et rectale - Implication pour la pratique clinique
}

\section{Conclusion}

\author{
G. Meurette \\ C Springer-Verlag France 2012
}

Les traumatismes anopérinéaux et rectaux se rencontrent dans des circonstances variées, mais toujours dans l'urgence. Ils peuvent impliquer plusieurs organes, avec la proximité de la filière gynécologique, urinaire et le cadre osseux pelvien. Un bilan clinique exhaustif est essentiel à leur prise en charge. Ce dernier sera réalisé au mieux et quasi systématiquement sous anesthésie générale pour se mettre dans des conditions idéales d'examen.

Le bilan complémentaire selon les circonstances du traumatisme peut comporter une imagerie et la tomodensitométrie représente l'examen le plus pertinent par les informations qu'il donne sur les lésions osseuses, pelviennes et vasculaires associées. La stratégie de prise en charge, souvent multidisciplinaire doit se faire dans l'urgence et ne saurait être différée que par l'existence de lésions vitales plus urgentes, car tout délai dans le traitement expose à des risques à court terme à la fois hémorragiques et infectieux, mais aussi à plus long terme sur le plan fonctionnel.

Après avoir mis le patient à l'abri d'un risque hémorragique vital (possible en cas de lésions vasculaires pelviennes) la réparation doit se faire de façon méthodique, en débutant par une stabilisation osseuse lorsqu'elle est nécessaire, puis une reconstitution des entités anatomiques avant que le champ des plaies ne soit largement modifié par l'oedeme réactionnel. Cette stratégie est très stéréotypée par les « 4D » que chacun doit bien garder présent à l'esprit dans sa prise en charge. Une fois passée la période aiguë, les séquelles fonctionnelles peuvent survenir. Il convient de bien en informer le patient quand se discutera la fermeture de la colostomie quand elle est confectionnée. Les facteurs prédictifs de séquelles sont surtout les délabrements sphinctériens et les blessures neurologiques concomitantes. Un suivi prolongé des patients sera nécessaire par assimilation ici aux traumatismes obstétricaux.

La situation postopératoire en chirurgie proctologique peut être source de complication que l'on peut rapprocher des traumatismes anorectaux. C'est ici par un suivi rapproché des patients opérés et un dépistage précoce des « suites opératoires anormales » qu'on évite les complications graves (septiques) et les séquelles.

Le cas particulier des traumatismes isolés du rectum possède ses spécificités. Les circonstances de survenue nécessitent d'être bien explicitées, surtout si un contexte médicolégal peut être mis en avant (agression sexuelle). La procédure de description des lésions et le bilan sont alors extrêmement importants. Une réparation des lésions souspéritonéales ne nécessitent pas toujours de dérivation digestive, mais une surveillance armée sera toujours la règle dans cette situation.

Une lésion mixte du rectum et de l'appareil sphinctérien doit faire raisonnablement opter dans la majorité des cas pour une stomie de dérivation associée à la réparation. 